\title{
Relación entre habilidades neuropsicológicas y comprensión lectora en Educación Primaria
}

\section{Relationship between neuropsychological skills and reading comprehension in Primary Education}

\author{
ISSN 1510-2432 - ISSN 1688-9304 (en línea) - DOI: 10.18861/cied.2017.8.2.2686
}

Rocío Carballar

Master en Neuropsicología y Educación, Universidad Internacional de la Rioja, España. Maestra de Educación Primaria, Universidad Complutense de Madrid. Docente del Taller de habilidades sociales, Asociación de atención en trastornos del espectro autista, SuresTEA, España.

Pilar Martín-Lobo

Doctora y Licenciada en Psicología, Universidad Complutense de Madrid, España. Licenciada en Filosofía y Letras. Investigadora en procesos neuropsicológicos del aprendizaje, neurociencia e idiomas, inteligencias múltiples y pizarra digital interactiva, talento y altas capacidades. Asesora internacional en aplicación de la neuropsicología a la educación. Profesora, Universidad Complutense de Madrid, Universidad Panamericana de México y la Escuela de Capacitación Pedagógica de Perú.

\begin{abstract}
A. Matías Gámez ${ }^{1}$
Doctor en Psicología, Universidad de Jaén, España. Especialización en Psicología experimental, aprendizaje humano y condicionamiento. Profesor de Procesos Psicológicos Básicos, Metodología de las Ciencias del Comportamiento y Psicología de la Educación. Investigador en contenidos del aprendizaje predictivo e instrumental humano y efectos de dependencia contextual en el aprendizaje instrumental.
\end{abstract}

Fecha de recibido: 22/02/2016

Fecha de aceptado: 21/08/2017

\section{Resumen}

La lectura es un proceso complejo en el que intervienen factores perceptivos, lingüísticos, cognitivos y neuropsicológicos. Algunos factores neuropsicológicos participan en el proceso lector desde su inicio, tales como los movimientos oculares que se realizan en la lectura, la motricidad y la lateralidad. El objetivo de este estudio fue evaluar la relación entre estos factores neuropsicológicos y la comprensión lectora en alumnos de Educación Primaria. Para ello se seleccionó una muestra de 52 niños de ocho años de edad en los que se evaluaron los movimientos oculares sacádicos para leer, los patrones motores básicos, la lateralidad y la comprensión lectora. Con los datos obtenidos se ha realizado un estudio descriptivo y correlacional cuyos resultados mostraron que existe una relación positiva y significativa entre las variables y que los alumnos con mejor habilidad de movimientos oculares sacádicos, mejor ejecución en patrones motores básicos y lateralidad homogénea obtuvieron mayores puntuaciones en comprensión lectora. Este estudio abre nuevas perspectivas teóricas y científicas para el aprendizaje de la lectura y la calidad educativa. Palabras clave: habilidades neuropsicológicas, comprensión lectora, patrones motores básicos, movimientos sacádicos, lateralidad. 


\begin{abstract}
Reading is a complex process which involves perceptual, linguistic, cognitive, and neuropsychological factors. Some neuropsychological factors are involved in the acquisition of the reading process such as eye movements that are carried out during the reading process, motor functions, and laterality. The aim of this study is the evaluation of the relationship between such neuropsychological factors and the reading comprehension process in Elementary Education students. A sample of 52 eight year old children was observed. Their saccadic movements while reading, basic motor patterns, laterality, and reading comprehension were evaluated. With the data obtained a descriptive and correlational study was carried out whose results showed that there is a positive and significant relationship between the abovesaid variables. It was also evidenced that those students with better execution of ocular saccadic movements, better execution of basic motor patterns and homogenous laterality showed a higher reading comprehension level. This study opens up new theoretical and scientific prospects for literacy learning and quality of education.
\end{abstract}

Key words: neuropsychological skills; reading comprehension; basic motor patterns; saccadic movements; laterality.

\title{
Introducción
}

El aprendizaje de la lectura influye en gran medida en el rendimiento escolar y en el futuro profesional de los alumnos ya que la lectura es una de las técnicas instrumentales básicas del aprendizaje. El porcentaje de alumnos con dificultades lectoras en la actualidad es bastante alto, convirtiéndose en muchas ocasiones en la causa y el origen del bajo rendimiento escolar (e.g., Dezcallar, Clariana, Cladellas, Badia y Gotzens, 2014; Gil, 2011; Kern y Friedman, 2009).

El informe PISA 2015 refleja la necesidad de mejorar la competencia lectora en España y en muchos otros países, por lo que es conveniente descubrir nuevos conocimientos relacionados con el funcionamiento del cerebro para leer y esto permitirá desarrollar propuestas de intervención que incidan directamente en aquellos aspectos implicados en la dificultad lectora.

En la lectura se realizan simultáneamente procesos perceptivos, léxicos, sintácticos y semánticos (Aragón-Jiménez, 2011) y el lector debe llevar a cabo una serie de operaciones cognitivas complejas (e.g., Cuetos, 2008). Los avances científicos actuales muestran las diferencias estructurales y funcionales del cerebro entre los buenos lectores y aquellos alumnos con dificultades lectoras. Por ejemplo, existen diferencias entre los disléxicos y no disléxicos que se han podido comprobar mediante técnicas de neuroimagen. La ruta funcional para la lectura activa diversos mecanismos cerebrales de los lóbulos parietal y occipital para la decodificación visual, el procesamiento fonológico y semántico y la comprensión, y del lóbulo frontal para la producción oral (Carboni-Román, Del Río Grande, Capilla, Maestú y Ortiz, 2006). Otras regiones cerebrales implicadas en la lectura son las áreas motoras y premotoras del córtex cerebral, los hemisferios cerebrales, el cerebelo y áreas subcorticales de los ganglios basales y el tálamo.

Estas áreas cerebrales han sido objeto de estudio en muchos trabajos como variables neuropsicológicas incidentes en el aprendizaje y en la lectura. Rosselli, Matute y Ardila (2006) realizaron un estudio en el que obtuvieron una correlación positiva entre la comprensión lectora y las pruebas neuropsicológicas de atención, habilidades construccionales y espaciales, memoria, lenguaje, habilidades metalingüísticas y funciones ejecutivas. Sin embargo, son escasos los estudios recientes dedicados a explorar la relación existente 
entre la comprensión lectora y habilidades neuropsicológicas relacionadas con la motricidad tales como los movimientos sacádicos, los patrones motores básicos y la lateralidad.

En el presente estudio se ha centrado la atención en los movimientos oculares relacionados con la lectura, en la motricidad y en la lateralidad para llevar a cabo los procesos lectores, los que implican numerosas áreas cerebrales (ver Tabla 1), áreas en las que se profundiza a continuación.

Tabla 1. Síntesis de las áreas cerebrales implicadas en la lectura

\begin{tabular}{|c|c|c|}
\hline & Áreas & Funciones \\
\hline & $\begin{array}{l}\text { Lóbulos temporal, } \\
\text { occipital, parietal y } \\
\text { frontal }\end{array}$ & $\begin{array}{l}\text { Decodificación visual, auditiva. } \\
\text { Procesamiento fonológico y semántico para la } \\
\text { comprensión. } \\
\text { Producción oral. }\end{array}$ \\
\hline & Circunvolución angular & $\begin{array}{l}\text { Asocia imagen visual de objetos y letras a imagen } \\
\text { auditiva de sus nombres. }\end{array}$ \\
\hline & Giro supramarginal & $\begin{array}{l}\text { Completa la decodificación dando significado total a } \\
\text { las frases. }\end{array}$ \\
\hline & Motora primaria & $\begin{array}{l}\text { Representación motora del cuerpo e inicio de } \\
\text { movimientos del órgano fonador. }\end{array}$ \\
\hline & Premotora & Controla los movimientos oculares. \\
\hline & Suplementaria & Iniciativa verbal y selección de movimientos. \\
\hline & $\begin{array}{l}\text { Perisilviana y parietal } \\
\text { del } \\
\text { hemisferio derecho }\end{array}$ & $\begin{array}{l}\text { Prosodia } \\
\text { Lenguaje con ritmo }\end{array}$ \\
\hline & Sensitiva primaria & Movimientos bucofaciales \\
\hline Subcortical & Cerebelo & Fluidez y articulación \\
\hline & Ganglios basales & $\begin{array}{l}\text { Regulación de la fluidez y coordinación de secuencias } \\
\text { motoras }\end{array}$ \\
\hline & Tálamo & $\begin{array}{l}\text { Conecta las áreas expresivas y comprensivas del } \\
\text { lenguaje }\end{array}$ \\
\hline
\end{tabular}

Fuente: Elaboración propia a partir de De la Peña (2016).

Las funciones visuales y auditivas y las habilidades fonológicas tienen un papel importante en el proceso de decodificación de la lectura (e.g., Rasinski, Blachowicz y Lems, 2012; Resnick y Weaver, 2010). Respecto a las funciones visuales, los movimientos oculares que se realizan durante la lectura -llamados movimientos sacádicos- son necesarios para leer, y si existe habilidad y buen control de estos movimientos, se mejora de forma significativa la fluidez lectora junto a los procesos cognitivos y la atención visoespacial (e.g., Leong et al., 2014). Los movimientos oculares se producen gracias a seis músculos regidos por los nervios craneales y, en concreto, los movimientos sacádicos son pequeños saltos que dan los ojos para poder pasar de un punto de fijación en el texto para captar la información a otro, y permiten a los ojos deslizarse por las palabras del texto. Cuando no hay buena motricidad ocular puede haber dificultades lectoras (Ferré y Aribau, 2002) y problemas de lectura en general por falta de habilidad de motilidad ocular, acomodación y visión binocular (Evans, 1998). Diferentes estudios coroborran estas afirmaciones, como el de López-Juez (2010) que ha señalado en sus trabajos que la motricidad ocular es una de las funciones visuales que más se ve alterada cuando surgen problemas de lectura. Otros han mostrado que una lectura deficiente se relaciona con una pobre ejecución en los test que evalúan los 
movimientos sacádicos (e.g., Miyata, Minagawa-Kawai, Watanabe, Sasaki y Ueda, 2012). En un estudio realizado recientemente para evaluar la influencia de distintas variables en el proceso lector se analizan los errores cometidos y el tiempo empleado en los movimientos sacádicos y se muestra que correlacionaban negativamente con la comprensión lectora, es decir, que una buena ejecución de los movimientos sacádicos se relacionaba con una buena comprensión lectora (Megino-Elvira, Martín-Lobo y Vergara, 2016).

En cuanto a los patrones motores básicos, autores de estudios de décadas anteriores señalaban que niños con carencias en los aspectos visomotores y visoperceptivos tenían el mismo nivel lector que otros niños sin ellas o que el entrenamiento perceptivo-visual y motor no incidía en el desarollo lector (e.g., Alegría, 1985; Delfior, 1993). Sin embargo, trabajos más recientes como el de Rigal (2006) muestran que los movimientos básicos facilitan el desarrollo motor y favorecen el aprendizaje de la lectura. Este autor explica que surgen como consecuencia de las transformaciones del sistema neuromuscular y evolucionan y aumentan su rendimiento con la edad. Por una parte, el movimiento del reptado, el gateo, la marcha, la carrera y el triscado se van adquiriendo a través de los movimientos de desplazamiento en el espacio del suelo que va realizando el bebé de forma natural. Un buen desarrollo motor favorece el aprendizaje de la lectura porque el movimiento del desplazamiento en el espacio favorece el movimiento motor y el movimiento ocular, al ir mirando la mano que avanza. Por ejemplo, en el reptado y en el gateo es donde el bebé ejercita el movimiento ocular y la convergencia visual junto a la coordinación visual y motriz y supone el inicio de habilidades que permiten mover los ojos para leer y mover la mano, el brazo y el sistema muscular para mantener la postura de la lectura y para escribir.

Por otra parte, los movimientos básicos facilitan y se relacionan con las habilidades perceptivo-motrices, la organización del espacio y del tiempo, así como de forma específica con los hábitos motores y neuromotores que inciden en la lectura de forma que para alcanzar un nivel de maduración lectora adecuada se requiere un nivel de desarrollo psicomotor, perceptivo, emocional y del lenguaje (e.g., Erazo, 2008). Asimismo, en la comprensión lectora intervienen otros factores de lenguaje, de la memoria y cognitivos que requieren una base neuropsicológica relacionada con la funcionalidad visual, auditiva, motriz y la lateralidad para que el niño pueda desarrollar su potencial cognitivo durante su etapa escolar (e.g., Anderson, 1994; López-Escribano, 2007).

Por último, la lateralidad hace referencia a la utilización preferente de un lado frente al otro del cuerpo (Harris, 1961) y es conveniente considerar el uso preferente de una mano sobre la otra, del ojo, del oído y del pie para valorar la lateralidad de un sujeto. Por ejemplo, si el sujeto es diestro utilizará la mano, el pie, el ojo y el oído del lado derecho, lo que permite afirmar que es una lateralidad homogénea. En el caso de que, por ejemplo, utilizara la mano, el oído y el pie del lado derecho pero el ojo dominante fuera el izquierdo, se puede decir que es una lateralidad no homogénea. En algunos estudios realizados en épocas anteriores como el de Longoni, Scalisi y Grilli (1989) no se hallaron diferencias significativas en cuanto al rendimiento de la lectura en niños con lateralidad cruzada, mientras que en otros trabajos hay evidencias de que la lateralidad se relaciona con la lectura (e.g., Allen y Wellman, 1980). De la misma manera, estudios más actuales también muestran que la lateralidad se relaciona con el aprendizaje y dominio de la competencia en la lectura. Por ejemplo, Mayolas, Villarroya y Reverter (2010) encontraron que los alumnos con lateralidad homogénea diestra obtenían mejores resultados en el aprendizaje de la lectura y que aquellos con cruce a nivel ocular y podal eran los que tenían mayores dificultades en la comprensión lectora, en organización y en atención en clase. 
Por todo lo expuesto se considera necesario profundizar en los procesos de lectura desde las aportaciones científicas actuales del funcionamiento cerebral en el proceso lector y de forma específica en las habilidades neuropsicológicas de los movimientos oculares para la lectura, los patrones básicos del movimiento, junto al tono muscular, al control postural y a la lateralidad para mejorar la comprensión lectora.

El objetivo general de este trabajo fue estudiar la relación existente entre las habilidades neuropsicológicas y la comprensión lectora. Los objetivos específicos fueron analizar las diferencias en habilidades de movimientos sacádicos, movimientos básicos y lateralidad, junto a la comprensión lectora y a la relación entre cada una de estas habilidades con el nivel de comprensión lectora en alumnos de Enseñanza Primaria. La hipótesis de partida es que existe una relación positiva entre los movimientos sacádicos, los patrones motores básicos y la lateralidad homogénea y el nivel de comprensión lectora en alumnos de siete y ocho años.

\section{Método}

\section{Participantes}

Para este estudio se seleccionó un total de 52 participantes (20 niñas y 32 varones) con edades comprendidas entre los siete y los ocho años a través de un procedimiento de muestreo incidental. Ninguno de los participantes tenía un Cl inferior a 70 o superior a 135, ni informes psicopedagógicos que reflejaran la presencia de trastornos de neurodesarrollo o deficiencias sensoriales. Todos ellos cursaban $3 .^{\circ}$ grado de Educación Primaria en un centro público de la Comunidad de Madrid con un estatus socioeconómico medio.

\section{Instrumentos}

Se llevó a cabo la evaluación de los movimientos sacádicos, los patrones motores básicos, la lateralidad y la comprensión lectora mediante la aplicación de los siguientes instrumentos de valoración:

- Test K-D de King y Devick (1976). La finalidad de esta prueba es valorar los movimientos sacádicos que realizan los ojos durante la lectura. El alumno tiene que leer una serie de números (que se disponen en varias tarjetas), mientras se anota el tiempo que tarda en leer la tarjeta y los errores cometidos. El test consta de cuatro tarjetas. La tarjeta de demostración es utilizada como ejemplo para que el alumno entienda cómo debe realizar el test correctamente. Cada número está unido por flechas para que el niño observe la dirección que debe tomar cuando proceda a la lectura de las otras tarjetas. La tarjeta I no dispone de flechas pero sí tiene líneas que unen los números. La tarjeta II no tiene líneas de unión entre los números y en la tarjeta III la separación vertical entre las líneas de números es menor. Asimismo, contiene tablas normativas para interpretar los resultados en función de la edad. En este estudio se han evaluado niños de siete y ocho años de modo que se considera que no superan la prueba los participantes de siete años que han invertido un tiempo superior a 100,89 segundos (con un margen de error de 25,16 segundos) y han cometido más de 11 errores. En cuanto a los niños de ocho años, no podrán exceder los 79,13 segundos (con margen de error de 27,35 segundos) y los tres errores. 
- Prueba de motricidad (adaptado del estudio de Santiuste, Martín-Lobo y Ayala, 2005). Esta prueba permite la valoración de diferentes patrones motores tales como arrastre, gateo, marcha, triscado, carrera, tono muscular y control postural. Se valora cada uno de ellos con una puntuación del 1 a 5 correspondiendo esta puntuación a los siguientes criterios: 1 (no supera severo), 2 (no supera alto), 3 (no supera medio), 4 (no supera leve) y 5 (supera).

- Adaptación del Test de Subirana (1952). Consiste en 40 ítems de aplicación individual que se corresponden con 40 acciones que la persona evaluada debe realizar. Está dividido en cuatro bloques de 10 ítems cada uno. Cada bloque se dedica a evaluar determinadas partes del cuerpo: ojo, mano, pie y oído. Una vez que se realiza la acción expresada en cada ítem se anota si se ha realizado con la derecha o con la izquierda, de modo que si se ejecutan más de seis acciones de un mismo bloque con la misma parte se considera que esta es parte predominante.

- Batería PROLEC (Cuetos, Rodríguez y Ruano, 1996). Esta prueba evalúa los procesos implicados en la lectura en niños de seis a diez años. Concretamente en este trabajo se empleó la prueba de comprensión de textos (procesos semánticos) en la que el alumno debe leer cuatro textos (dos de tipo expositivo y dos de tipo narrativo) y responder a cuatro preguntas por cada texto. Por cada respuesta correcta obtenida se asigna un punto de modo que el cómputo total (máximo 16) es transformado en percentiles.

\section{Procedimiento}

Una vez obtenida la aprobación del centro educativo y la autorización de los padres de los participantes se procedió a la aplicación de las pruebas de evaluación por personal especializado y de manera individualizada. Durante las dos primeras sesiones de evaluación se aplicó la prueba de motricidad. La puntuación de cada participante en esta prueba se valoró por la media de las puntuaciones obtenidas en cada patrón motor. En las siguientes sesiones se aplicó la prueba de lateralidad y el test K-D, todos de forma individual en un aula vacía para evitar distracciones. La última sesión de evaluación se dedicó a la aplicación de la prueba de comprensión lectora que se realizó conjuntamente en la misma aula. Cada participante recibió los textos con sus correspondientes preguntas y hasta que no finalizaban todos los alumnos no se repartía el siguiente texto.

\section{Resultados}

En primer lugar se llevó a cabo un análisis descriptivo de los resultados de la evaluación de cada una de las variables implicadas en este trabajo y a continuación se calcularon los coeficientes de correlación correspondientes para conocer las correlaciones existentes entre esas variables.

\section{Análisis descriptivo}

En cuanto a los resultados de los movimientos oculares sacádicos se encontró que el test K-D fue superado por un $61.5 \%$ de los participantes frente al $38.5 \%$ que no lo superaron, lo cual evidencia las dificultades de movimientos oculares que mostraba este porcentaje de alumnos para leer de forma eficiente. En la Tabla 2 se presenta el análisis descriptivo relativo al tiempo empleado y a los errores cometidos en esta prueba. 
Tabla 2. Análisis descriptivo de la variable movimientos oculares sacádicos

\begin{tabular}{ccc} 
& Tiempo total & Errores totales \\
\hline Media & 97,56 & 1,90 \\
\hline Desv. Típica & 25,60 & 3,87 \\
\hline Máximo & 135,17 & 15,00 \\
\hline Mínimo & 69,95 & 0,00 \\
\hline
\end{tabular}

Respecto a los resultados de la prueba de patrones motores básicos la puntación mínima obtenida en todos los patrones motores ha sido de 3 puntos (no supera medio) y la máxima de 5 puntos (supera). Ningún alumno obtuvo puntuaciones por debajo de 3.

En la Tabla 3 se muestra la puntuación media obtenida en cada patrón motor. Como puede observarse, el gateo, la marcha y la carrera han sido los mejor ejecutados por los alumnos obteniendo una media de 4,58 en los tres patrones. Sin embargo, el arrastre y el triscado han sido los patrones peor ejecutados obteniendo una media de 4,10 y 3,83 respectivamente. El tono muscular y el control postural se mantienen por encima del triscado y el reptado o arrastre con una media de 4,25 y de 4,38 respectivamente.

Tabla 3. Análisis descriptivo de la variable patrones motores básicos

\begin{tabular}{cccccccc} 
& Arrastre & Gateo & Marcha & Carrera & Triscado & $\begin{array}{c}\text { Tono } \\
\text { muscular }\end{array}$ & $\begin{array}{c}\text { Control } \\
\text { postural }\end{array}$ \\
\hline Media & 4,10 & 4,58 & 4,58 & 4,58 & 3,83 & 4,25 & 4,38 \\
\hline Desv. típica & 0,80 & 0,61 & 0,64 & 0,64 & 0,81 & 0,65 & 0,69 \\
\hline Máximo & 5,00 & 5,00 & 5,00 & 5,00 & 5,00 & 5,00 & 5,00 \\
\hline Mínimo & 3,00 & 3,00 & 3,00 & 3,00 & 3,00 & 3,00 & 3,00 \\
\hline
\end{tabular}

En cuanto a la variable lateralidad, tal y como puede apreciarse en la Tabla 4 el mayor número de alumnos son diestros (53.8\%). Por su parte, solo el $9.6 \%$ de los alumnos de la muestra resultaron zurdos mientras que el resto (36.6\%) tenían lateralidad cruzada. Para el cálculo posterior de la correlación entre esta variable y la comprensión lectora la lateralidad se ha categorizado en dos grandes grupos: lateralidad homogénea, que incluye a los alumnos diestros y zurdos, y lateralidad heterogénea, que contiene el resto de alumnos. Se obtuvo de esta manera un mayor porcentaje de alumnos con lateralidad homogénea (63.5\%) que con lateralidad heterogénea (36.5\%).

Tabla 4. Porcentaje de cada tipo de lateralidad

\begin{tabular}{|l|c|}
\hline Diestro & 53.8 \\
\hline Lateralidad cruzada & 36.6 \\
\hline Zurdo & 9.6 \\
\hline
\end{tabular}

Por último, en relación con los resultados de la prueba de comprensión lectora, la Tabla 5 muestra que la puntuación media de los alumnos de la muestra en la prueba de comprensión de textos del PROLEC fue de 12,25. Asimismo es necesario hacer notar que el $19.2 \%$ de los alumnos obtuvieron la puntuación máxima mientras que solo el 3.8\% respondieron 6 respuestas correctas, lo que corresponde a la puntuación mínima obtenida por la muestra. 
Tabla 5. Análisis descriptivo de la variable comprensión lectora

\begin{tabular}{ll}
\hline Media & 12,25 \\
\hline Desviación típica & 3,04 \\
\hline Máximo & 16,00 \\
\hline Mínimo & 6,00 \\
\hline
\end{tabular}

\section{Análisis correlacional}

Se calculó el coeficiente de correlación de Pearson para evaluar la relación entre los movimientos sacádicos y los patrones motores básicos con la comprensión lectora. Por su parte, al tratarse la lateralidad de una variable dicotómica, para explorar la relación entre esta y la comprensión lectora se utilizó el coeficiente de correlación biserial-puntual. En cualquier caso, se realizaron pruebas de significación estadística para cada coeficiente con un criterio de rechazo de $p<.05$.

La Tabla 6 muestra la matriz de correlaciones realizadas en este estudio, donde se exponen los resultados obtenidos en los análisis de correlación entre movimientos oculares, patrones motores básicos y lateralidad y la comprensión lectora. Se observa que existe una correlación estadísticamente significativa entre cada una de las variables analizadas y la comprensión lectora.

Tabla 6. Matriz de correlaciones

\begin{tabular}{|l|l|l|}
\hline \multicolumn{2}{|l|}{} & Comprensión lectora \\
\hline Movimientos oculares & Pearson &, $877^{* *}$ \\
& Sig. &, 000 \\
\hline Patrones motores básicos & Pearson &, $453^{* *}$ \\
& Sig. &, 001 \\
\hline Lateralidad & Biserial-puntual &, $592^{* *}$ \\
& Sig. &, 000 \\
\hline
\end{tabular}

En primer lugar, por una parte la correlación entre la ejecución de los movimientos oculares sacádicos y el nivel de comprensión lectora fue positiva, lo que implica que a mejor ejecución en los movimientos sacádicos mayor nivel de comprensión lectora. Por otra parte, la correlación entre patrones motores básicos y comprensión lectora también resultó positiva, lo que significa que a mejor ejecución en la prueba de patrones motores básicos mayor nivel de comprensión lectora. Por último, el que la correlación entre lateralidad y comprensión lectora también sea positiva debe interpretarse, a la vista de los datos, como que los alumnos con lateralidad homogénea presentan un nivel mayor de comprensión lectora. En definitiva podemos decir que una buena ejecución de los movimientos oculares sacádicos y de los patrones motores básicos y una lateralidad homogénea se relacionan con una buena comprensión lectora.

\section{Discusión}

El objetivo de este trabajo fue evaluar la relación entre los movimientos sacádicos, los patrones motores básicos, la lateralidad y la comprensión lectora, en alumnos de segundo ciclo de Educación Primaria. Los resultados de los análisis correlacionales han puesto de manifiesto que variables relacionadas con la motricidad tienen relación con la comprensión 
lectora. Este resultado está en consonancia con la idea de que para una buena comprensión lectora es necesario un buen desarrollo de los componentes cognitivo, neuropsicológico y optométrico del niño (e.g., Cuetos, 2008; Martín-Lobo, 2003).

Respecto a los movimientos sacádicos, los resultados indican que existe una relación positiva entre ambas variables. De hecho, los datos obtenidos por los autores señalan que los alumnos que no consiguen realizar la prueba correctamente son los que obtienen las puntaciones más bajas en la prueba de comprensión lectora. Esto resulta lógico teniendo en cuenta que una buena ejecución de los movimientos sacádicos se relaciona con la velocidad lectora, y esta con la comprensión del texto leído. El presente resultado está en la misma linea de los resultados de estudios previos en los que se ha puesto de manifiesto que las personas con un bajo rendimiento lector muestran deficiencias en los movimientos sacádicos, como se recoge en estudios como el de López-Juez (2010) que explica la relación existente entre el grado de disfunción en los movimientos oculares y las dificultades de comprensión lectora (e.g., Ferré y Aribau, 2002; Megino-Elvira et al., 2016).

En cuanto a los patrones motores básicos, los resultados de este trabajo muestran una relación positiva entre la ejecución motriz y la comprensión lectora. Es decir, una mejor ejecución motriz se relaciona con mayores niveles de comprensión lectora. Algunos autores han apuntado previamente que es conveniente un correcto desarrollo de estos patrones motores para favorecer el aprendizaje de la lectura (e.g., Rigal, 2006). Lo que resulta más novedoso es que estos patrones se relacionen específicamente con la comprensión lectora.

Por último, en relación con la lateralidad se ha encontrado que una lateralidad homogénea está en relación con una mejor ejecución en la prueba de comprensión lectora, lo que resulta coherente con otros estudios que demuestran la influencia de la lateralidad en los procesos de lectura y como consecuencia en las alteraciones de la misma (e.g., Mayolas et al., 2010). Un análisis más detallado de los datos obtenidos en estas pruebas permite observar que los alumnos con menos respuestas correctas en la prueba de comprensión lectora coinciden en gran medida con los alumnos con una lateralidad heterogénea, por lo que se obtienen resultados en consonancia con los de Mayolas et al. (2010).

Tomados en conjunto, los resultados obtenidos en este estudio suponen un apoyo a aquellos trabajos sobre el rendimiento escolar que postulan que el desarrollo motor es fundamental para el aprendizaje (e.g., Erazo, 2008; Martín-Lobo, 2003). Estos autores señalan que la motricidad es uno de los aspectos que influyen en los procesos de desarrollo y aprendizaje de la lectura, ya que diferentes áreas motrices se ven implicadas en los mecanismos de la misma. En definitiva, los resultados de este estudio arrojan un poco de luz sobre los factores relacionados con una variable tan importante para el aprendizaje y, por tanto, para el rendimiento escolar como es la comprensión lectora. Será necesario llevar a cabo nuevas investigaciones para seguir indagando cuáles son estos factores y cómo interaccionan entre ellos, con el objetivo de poder diseñar intervenciones eficaces para lograr mejorar la comprensión lectora y, por extensión, el rendimiento académico.

Las limitaciones de este trabajo se pueden considerar en cuanto al número de participantes ya que podría haber sido una muestra más amplia para dar mayor validez al trabajo.

Este estudio abre nuevas vías de investigación para el futuro, de forma que podrían realizarse trabajos similares en otras edades escolares, desde los 6 años hasta los 12 en la etapa en la que se consolida el dominio lector, así como diseñar, desarrollar y aplicar un programa de mejora de la lectura incluyendo la mejora de las habilidades neuropsicológicas 
y un plan de formación de profesores que puedan aplicar la neuropsicología en el ámbito educativo con la preparación y el nivel de innovación que estos programas de intervención requieren.

\section{Referencias bibliográficas}

Alegría, J. (1985). Por un enfoque psicolingüístico del aprendizaje de la lectura y sus dificultades. Infancia y aprendizaje, 29, 79-94.

Allen, M. y Wellman, M.W. (1980). Hand position during writing, cerebral laterality and reading: age and sex differences. Neuropsychologia, 18, 33-40.

Anderson, R.C. (1994). Role of the reader's schema in comprehension, learning, and memory. En R.B. Ruddell, M.R. Ruddell \& H. Singer (Eds.), Theoretical Models and Processes of Reading. Newark, DE: International Reading Association.

Aragón-Jiménez, V. (2011). Procesos implicados en la lectura. Innovación y experiencias educativas, 39, 1-11.

Carboni-Román, A., Del Río Grande, D., Capilla, A., Maestú, F. y Ortiz, T. (2006). Bases neurobiológicas de las dificultades de aprendizaje. Neurología, 42(S2), S171-S175.

Cuetos, F. (2008). Psicología de la lectura. Madrid: Wolters Kluwer Educación.

Cuetos, F., Rodríguez, B. y Ruano, E. (1996). Evaluación de los procesos lectores PROLEC. Madrid: TEA ediciones.

De la Peña, C. (2016). Programas para la dislexia desde la base neuropsicológica. En P. Martín-Lobo (Coord.), Procesos y programas de neuropsicología educativa (pp. 169-177). España: Centro Nacional de Investigación e Innovación (CNIIE).

Delfior, S. (1993). Las dificultades de lectura: papel que juegan las deficiencias de lenguaje. Comunicación, lenguaje y educación, 17, 3-13.

Dezcallar, T., Clariana, M., Cladellas, R., Badia, M. y Gotzens, C. (2014). La lectura por placer: su incidencia en el rendimiento académico, las horas de televisión y las horas de videojuegos. Ocnos, 12, 107-116.

Erazo, N. (2008). La educación psicomotriz en el ciclo inicial como base para facilitar el aprendizaje de la lectura y escritura en niños y niñas. Universidad de Cádiz, España.

Evans, B. (1998). The underachieving child. Ophthalmic and Physiological Optic, 18, 153-159.

Ferré, J. y Aribau, E. (2002). El desarrollo neurofuncional del niño y sus trastornos. Visión, aprendizaje y otras funciones cognitivas. Barcelona: Lebón.

Gil, J. (2011). Hábitos lectores y competencias básicas en el alumnado de Educación Secundaria Obligatoria. Educación XXI, 14, 117-134.

Harris, A.J. (1961). Manuel d'application des tests de latéralité. París: C.P.A. 
Kern, M.L. y Friedman, H.S. (2009). Early educational milestones as predictors of lifelong academic achievement, midlife adjustment, and longevity. Journal of Applied Developmental Psychology, 30, 419-430.

King, A.T. y Devick, S. (1976). The proposed King-Devick test and its relation to the Pierce saccade test and reading levels. Chicago: Illinois College of Optometry.

Leong, D.F., Master, C.L., Messner, L.V., Pang, Y., Smith, C. y Starling, A.J. (2014). The effect of saccadic training on early reading fluency. Clinical pediatrics, 53, 858-864.

Longoni, A.M., Scalisi, T.G. y Grilli, M. (1989). Lateral preference and verbal skills: a survey in school settings. International Journal of Neuroscience, 44, 41-52.

López-Escribano, C. (2007). Contribuciones de la neurociencia al diagnóstico y tratamiento educativo de la dislexia del desarrollo. Revista Neurología, 44, 173-180.

López-Juez, M.J. (2010). ¿Por qué yo no puedo? Fundamentos biólogicos de las dificultades de aprendizaje. Madrid: Autor.

Martín-Lobo, M.P. (2003). La lectura. Procesos neuropsicológicos del aprendizaje, diagnósticos, estudio de casos y programas de intervención. Barcelona: Lebón.

Mayolas, M.C., Villarroya, A. y Reverter, J. (2010). Relación entre la lateralidad y los aprendizajes escolares. Apunts. Educación Física y Deportes, 101, 32-42.

Megino-Elvira, L., Martín-Lobo, M.P. y Vergara-Moragues, E. (2016). Influence of eye movements, auditory perception, and phonemic awareness in the reading process. The Journal of Educational Research, 109, 567-573.

Miyata, H., Minagawa-Kawai, Y., Watanabe, S., Sasaki, T. y Ueda, K. (2012). Reading speed, comprehension and eye movements while reading Japanese novels: Evidence from untrained readers and cases of speed-reading trainees. PloS One, 7(5), e36091.

Rasinski, T., Blachowicz, C. y Lems, K. (eds.) (2012). Fluency instruction. Research-based best practices. New York, NY: The Guilford Press.

Resnick, L.B. y Weaver, P.A. (2010). Theory and practice of early reading. New York: Routledge.

Rigal, R. (2006). Educación motriz y educación psicomotriz en preescolar y primaria. Barcelona: INDE Publicaciones.

Rosselli, M., Matute, E., y Ardila, A. (2006). Neuropsychological predictors of reading ability in Spanish. Revista Neurología, 42, 202-210.

Santiuste, V., Martín-Lobo, M.P. y Ayala, C. (2005). Bases neuropsicológicas del fracaso escolar. Madrid: Fugaz.

Subirana, A. (1952). La droiterie. Schweizer Archiv Fur Neurologie Und Psychiatrie, 69, 321-359.

\section{(Endnotes)}

${ }^{1}$ Este autor se encuentra actualmente en la Universidad de Cádiz, España. 